EPiC Series in Engineering
Volume 3, 2018, Pages 266-273
HIC 2018. 13th International
Conference on Hydroinformatics

\title{
Optimal time step length to minimize uncertainty of zonal water balance calculation in drinking water distribution systems
}

\author{
Attila Bibok ${ }^{1}$, Roland Fülöp ${ }^{1}$ \\ ${ }^{1}$ Budapest University of Technology and Economics, Müegyetem rkp $3 \mathrm{kmf} 45$, \\ Budapest H-1111, Hungary \\ Corresponding author: bibok.attila@epito.bme.hu
}

\begin{abstract}
Water balance calculation is a well-known and adapted method to analyze district-metered areas (DMAs) of drinking water distribution system. Other application of water balance results can be training dataset for consumption forecast, monitoring and managing water loss. The vast amount of application makes it a very powerful tool, which is in contrary sensitive to the accuracy of the calculation. The uncertainty of flow metering decreases as the length of the time step decreases, however, the uncertainty of stored volume measurement in tanks increases. Investigation of the limitations of water balance calculation in regard to uncertainty is necessary to develop an analytical solution for optimal calculation time step.
\end{abstract}

\section{Introduction}

Zonal water balance analysis is capable of monitoring and quantifying water losses [1]. Common calculation time-step lengths are 30 or 60 minutes $[1,2,9]$. Minimum night flow is one of the main indicators of water loss on a distribution system [3], which can be determined as the minimum of water consumption pattern $[4,9]$. The lower the time step length, the bigger the resolution of the consumption pattern. However, by decreasing the time step length, some obviously false result can occur, like negative consumption. The calculated minimum night flow depends on the resolution of the water balance (Figure 1). The difference can be as high as 60\%. Minimum night flow analysis relies on zonal water balance calculations, which makes it essential to provide as accurate water balance calculations as possible. Water metering in low flow regime is problematic because as the flow rate decreases, the uncertainty of the flow metering increases [5]. Most of the pump schedule optimization methods use short-term water consumption forecast, which is sensitive to the accuracy of the historic water consumption values [11]. Therefore, the uncertainty of zonal water balance calculation affects most of the real-time pump-schedule optimization methods either. 
Optimal Time Step Length to Minimize Uncertainty of Zonal Water Balance ...A.Bibok and R. Fülöp

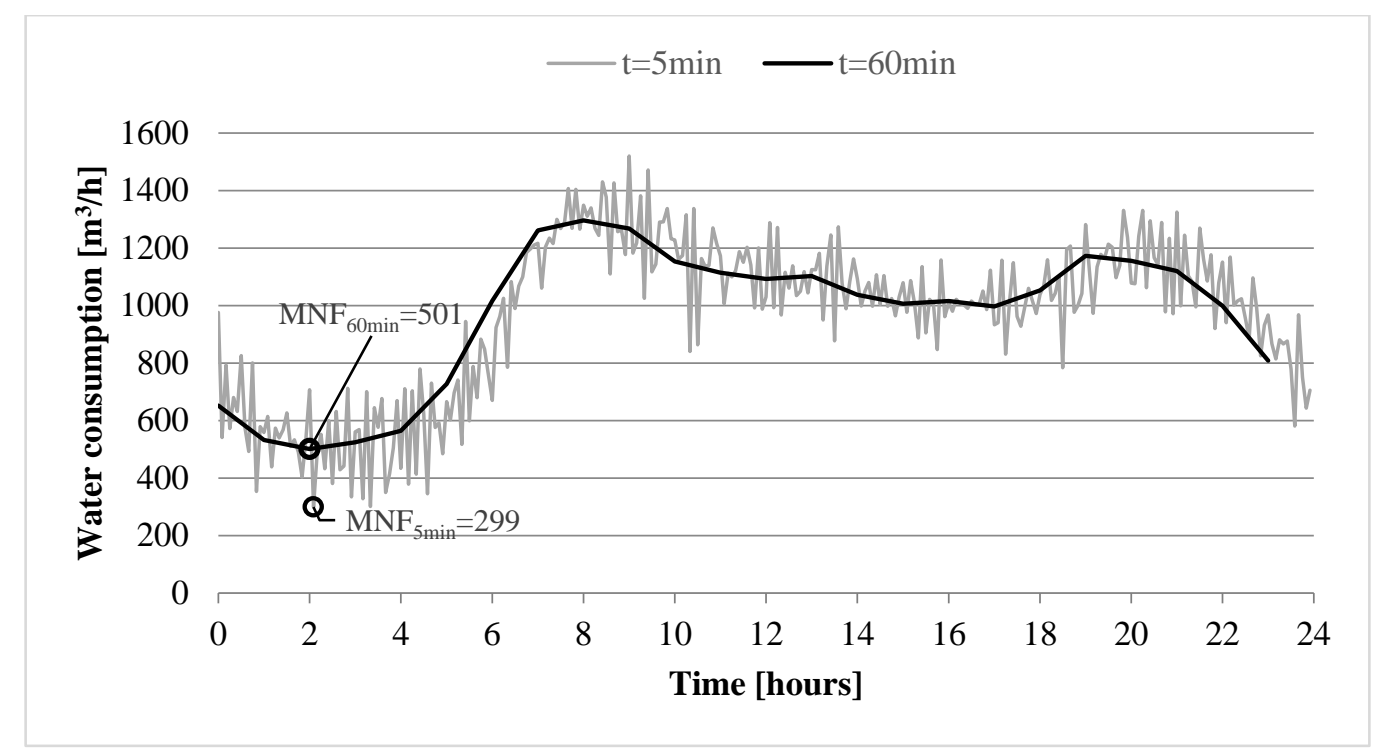

Figure 1 Minimum night flow calculated by different time step length

\section{Methodology}

Zonal water balance calculations are valid if all the following values are measured:

- Flow inputs/outputs of the zone

- $\quad$ Stored flow in the tanks of the zone

The stored flow can be calculated by the difference of two stored volume measurements if there is lack of direct flow measurement on the supply pipes of the tanks.

$$
Q_{t}=\sum_{i=1}^{n} Q_{i n, i, t}-\sum_{j=1}^{m} Q_{o u t, j, t}-\sum_{k=1}^{p}\left(V_{k, t}-V_{k, t-1}\right)
$$

Where $Q_{t}$ is the flow rate at timestep $t, Q_{i n, i, t}$ is the input flow rate at timestep $t Q_{o u t, j, t}$ is the output flow rate at timestep $t, V_{k, t}$ is the stored volume at the end of timestep $t V_{k, t-1}$ is the stored volume at the beginning of timestep $t$.

Flow and level measurements are results of instantaneous sampling in this study. No moving average calculation or outlier detection was performed on the datasets. If a DMA does not contain storage tanks, or measured output, these components of equation (1) are equal to 0 . The equation for zonal water balance is still valid in these situations.

\subsubsection{Input, output flows}

If a pump starts between the end and the start of the time step, the volume pumped until the next measurement is not included in the calculation. On the other hand, if a pump stops between the end and the start of the time step, the calculated flow for the time step is going to be higher than in reality. Equal distribution of pump start and stop events was assumed, thus $1 / 2$ of the nominal flow rate is the expected value of uncertainty.

$u_{q}=\frac{Q^{*} t}{2 * 60 \min }$ 
Where $u_{q}$ is the uncertainty of input/output flow $\left[\mathrm{m}^{3} / \mathrm{h}\right]$, Q is the nominal flow rate of the pump in $\mathrm{m}^{3} / \mathrm{h}$, $\mathrm{t}$ is the time step in [min]

It is conventional to characterize flow metering accuracy by a percentage of reading or by a percentage of a full scale. Although different types of flow meters have different characteristics of uncertainty over different domains of the flow range. Water distribution mains usually have a diameter of $150 \mathrm{~mm}$ to 1600 depending on the layout of the system. Hungarian water utilities conventionally install ultrasonic or electromagnetic flow meters in their mains.

According to the specifications of various manufacturers, the uncertainty of the meters are 0,5 to $1,0 \%$ of the full scale [6], but MID certifications contain more information about the actual limitations of the accuracy of the meters at low flow conditions [6],[7]. The maximum velocity that is used to determine the full scale is almost an order higher than the maximum velocity of $2,0 \mathrm{~m} / \mathrm{s}$ that is usually observed in the mains. The uncertainty of measurement increases as the velocity decreases under $0,1 \mathrm{~m} / \mathrm{s}$. This phenomenon can be observed in case of extremely low flows $(<0,05 \mathrm{~m} / \mathrm{s})$, which makes water balance calculations ever more problematic at the time of minimum night flow. The same phenomenon is also present when small diameter domestic consumption meters face extreme low flow rates [5].

According to Measurement Instrument Directive (MID) 2014/32/EU Annex III (MI-001) the maximum permissible error (MPE) is $5 \%$ between the Minimum flowrate (Q1) and the Transitional flowrate (Q2) [8]. The MPE is $2 \%$ between Q2 and Overload flowrate (Q4). Q1-Q4 characteristic flow values vary by manufacturer, product, and diameter. The adjusted uncertainty value of flow rate calculation gives a more accurate estimation than equation (2), but it requires more information about the actual flow meters.

$u_{q}^{*}=\frac{Q^{*} t}{2 * 60 \min } *(1+\operatorname{MPE}(Q))$

Where $\mathrm{u}_{\mathrm{q}}{ }^{*}$ is the uncertainty of input/output flow $\left[\mathrm{m}^{3} / \mathrm{h}\right]$, Q is the nominal flow rate as the function of flow velocity $\mathrm{m}^{3} / \mathrm{h}, \mathrm{t}$ is the time step in [min].

The comparison of Q1 values of ultrasonic and electromagnetic meters shows that the minimum flow rate value from which the measurements

Table 1: Comparison of ultrasonic and electromagnetic flow meters

\begin{tabular}{lcccccc}
\hline \multirow{2}{*}{$\begin{array}{c}\text { Diameter } \\
{[\mathrm{mm}]}\end{array}$} & \multicolumn{2}{c}{ Ultrasonic } & \multicolumn{2}{c}{ Electromagnetic I } & \multicolumn{2}{c}{ Electromagnetic II } \\
\cline { 2 - 7 } & $\mathrm{Q}_{1}\left[\mathrm{~m}^{3} / \mathrm{h}\right]$ & $\mathrm{v}_{1}[\mathrm{~m} / \mathrm{s}]$ & $\mathrm{Q}_{1}\left[\mathrm{~m}^{3} / \mathrm{h}\right]$ & $\mathrm{v}_{1}[\mathrm{~m} / \mathrm{s}]$ & $\mathrm{Q}_{1}\left[\mathrm{~m}^{3} / \mathrm{h}\right]$ & $\mathrm{v}_{1}[\mathrm{~m} / \mathrm{s}]$ \\
\hline 80 & 0,80 & 0,0003 & 0,25 & 0,009 & 0,32 & \\
100 & 1,25 & 0,027 & 0,39 & 0,009 & 0,5 & \\
150 & 3,13 & 0,030 & 0,63 & 0,006 & 1,26 & \\
200 & 5,00 & 0,027 & 1,00 & 0,005 & 2 & \\
250 & 12,50 & 0,044 & 1,60 & 0,006 & 3,2 & \\
300 & 12,50 & 0,030 & 2,50 & 0,006 & 5 & \\
\hline
\end{tabular}

Any disturbance in the fully developed flow results in decreased accuracy. Fully bore electromagnetic meters are less sensitive to flow disturbances than insertion-type electromagnetic flow meters.

MID Classification was used for comparison, although newer standards exist, because most of the flow meters, which are installed in the system of Budapest Waterworks had this certificate in common. Different flow meter classification standards exist, depending on the region the meter is going to be distributed: 
- ISO 4064-1:2014: It is issued by the International Organization for Standardization. The conditions and requirements for the measurements are detailed for both electrical and mechanical flow meters.

- EN 14154: It is in compliance with ISO 4064-2:2014, issued by the European Committee for Standardization

- OIML R49: It is in compliance with ISO 4064-2:2014, issued by the International Organization of Legal Metrology.

- AWWA C7xx-15: The American Water Works Association differentiate the standards by the type and mechanical or physical principals the meters utilize. Every technology has its specific requirements.

\subsubsection{Stored flow}

The water volume stored in a tank is calculated from the water level measurement multiplied by the water surface area. If the wall of the tank is not vertical, which means the water surface area is the function of water level, the uncertainty of volume calculation changes as a function of the water level. Thus, the uncertainty of stored flow changes as well, which is calculated by the difference of stored volume at the start and at the end of the time step.

$u_{\text {tank }}=\frac{A(h) * u_{w l} * 60_{\min }}{t}$

Where: $\mathrm{u}_{\text {tank }}$ is the uncertainty of stored flow in $\left[\mathrm{m}^{3} / \mathrm{h}\right]$, A is the water surface area in $\left[\mathrm{m}^{2}\right]$, $\mathrm{h}$ is the water level in [m], $\mathrm{u}_{\mathrm{wl}}$ is the uncertainty of water level measurement in [m], $\mathrm{t}$ is the time step in [min]

There are various types of level meters, but the most widespread is ultrasonic and radar-based of noncontact types and hydrostatic pressure meters of contact type. Hydrostatic pressure has an accuracy of $+-0,25 \%$ of the full scale, which means $+-7,0$ centimeters for a 0-50 PSIG sensor. This level of accuracy is critical when a backpressure tank with large surface area supplies a zone with low water demand, that makes minimum night flow calculations problematic. Radar sensors are one order more accurate compared to pressure based level sensors in the scale of drinking water storage tanks. These sensors have $+-2-5 \mathrm{~mm}$ accuracy for the whole range.

\subsection{Optimal calculation time step}

The uncertainty of the water balance is the combination of the uncertainties of stored flow calculation of tanks and flow rate measurements of input and outputs regarding the actual zone of the distribution network. The uncertainty of stored flow calculation (Equation 4) increases as the time step length decreases, however flow rate measurement's uncertainty increases in this case. Because of this phenomenon, uncertainty of water balance always have a minimum, when time step is a positive real number (Figure 2). Combined uncertainty is calculated as the combination of the uncertainty of every input/output using equation (2) and stored flow data using equation (4). 
Optimal Time Step Length to Minimize Uncertainty of Zonal Water Balance ...A.Bibok and R. Fülöp

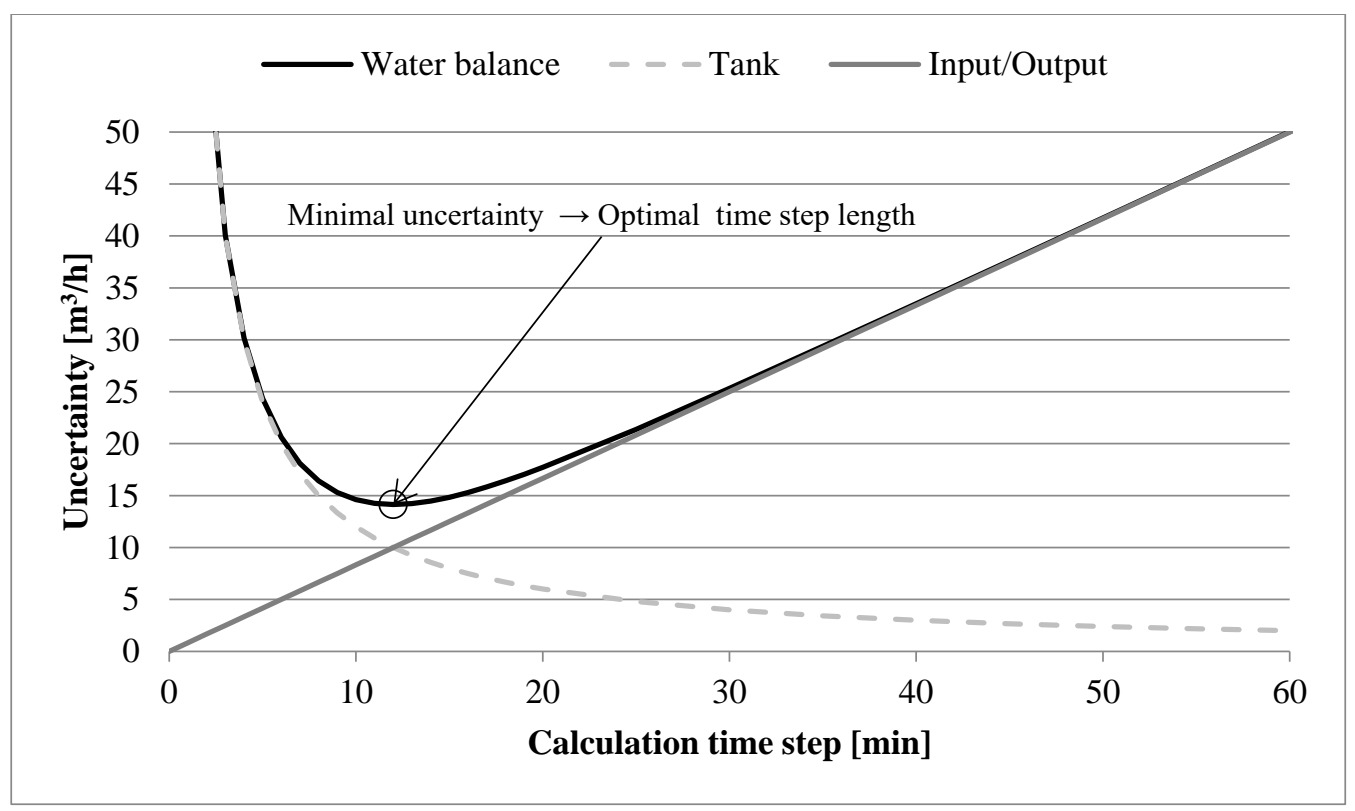

Figure 2 Uncertainty of water balance as a function of calculation time step

The following equation describes the uncertainty of zonal water balance calculation:

$u_{w b}(t)=\sqrt{\sum_{j=1}^{m}\left(\frac{Q_{j} t}{2 * 60 \min }\right)^{2}+\sum_{i=1}^{n} 2\left(\frac{A_{i} u_{w l, i} * 60 \mathrm{~min}}{t}\right)^{2}}$

Where: $u_{w b}$ is the uncertainty of water balance in $\left[\mathrm{m}^{3} / \mathrm{h}\right], \mathrm{t}$ is the calculation time step in [min], $\mathrm{Q}_{\mathrm{j}}$ is the nominal flow rate of pump $\mathrm{j}\left[\mathrm{m}^{3} / \mathrm{h}\right], \mathrm{A}$ is the area of water surface in $\left[\mathrm{m}^{2}\right], \mathrm{u}_{\mathrm{wl}, \mathrm{i}}$ is the uncertainty of water level measurement of tank i in [m]

Using an analytical approach, the optimal time step equals the root of the derivative of $\mathrm{u}_{\mathrm{wb}}(\mathrm{t})$ :

$$
t=\frac{\sqrt[4]{\sum_{i=1}^{n} 2\left(A_{i} u_{w l, i} * 60 \mathrm{~min}\right)^{2}}}{\sqrt[4]{\sum_{j=1}^{m}\left(\frac{Q_{j}}{2 * 60 \mathrm{~min}}\right)^{2}}}
$$

Where $\mathrm{t}>0$

The explicit Equation (6) of optimal time step length contains only the properties of the actual zone or DMA. If a zone has no storage tank, and variable speed pumps pressurize it, equation 9 and 10 are still valid, but the result for $t$ is going to be 0 minute. This is true in theory, but every flow meter has a maximum sampling rate, which is going to be the optimal time step in that case. If the sampling would have been based on integral flow meter readings, the uncertainty equation for flow metering would not have been valid, because the flow rate value would represent the mean flow rate instead of instantaneous sampling. 


\subsection{Pilot zones}

Six zones were investigated using equation (6) to see, how the flow rate of the pumps, and the surface of the tanks alter the optimal calculation time step. All of the zones are located in the distribution network of Budapest Waterworks. Some of the zones are on the periphery of the distribution network, but some of them has transmission function too. The uncertainty of tank level measurement was equally $0.01 \mathrm{~m}$. Although some of the water level meters had lower uncertainty values, but the data was stored in the SCADA system in meters using two digits resolution. Most of the zones had more than one tanks or chambers with independent level measurements. By disconnecting some of the chambers from the zone, it is possible to decrease time step length or water balance uncertainty. These modified time step lengths are displayed in column t' of Table 2.

\section{Results and discussion}

The optimal time step length for zonal water balance calculations depends on the properties of the tanks and pumps connected to the zone. The equation given is valid for constant surface area tanks. If the zone in question does not meet this criterion, the surface area has to be calculated as a function of water level in the tank. Based on the analysis of six different zones, the optimal time step varies between 6 and 33 minutes. The modified timestep length had a range of 7 to 23 minutes.

Table 2 Optimal time step for the pilot zones

\begin{tabular}{cccccc}
\hline Zone & $\mathrm{Q}\left[\mathrm{m}^{3} / \mathrm{h}\right]$ & $\mathrm{A}\left[\mathrm{m}^{2}\right]$ & $\mathrm{u}_{\mathrm{wl}}[\mathrm{m}]$ & $\mathrm{t}[\mathrm{min}]$ & $\mathrm{t}^{\prime}[\mathrm{min}]$ \\
\hline Zone 1 & $70 ; 32 ; 8$ & $30 ; 16$ & 0.01 & 7 & 6 \\
Zone 2 & $800 ; 600$ & $377 ; 616 ; 616$ & 0.01 & 10 & 6 \\
Zone 3 & 25 & 20 & 0.01 & 9 & 9 \\
Zone 4 & 8 & $30 ; 30$ & 0.01 & 23 & 20 \\
Zone 5 & 32 & $25 ; 25$ & 0.01 & 11 & 9 \\
Zone 6 & $450 ; 130$ & $200 ; 200$ & 0.01 & 8 & 7 \\
\hline
\end{tabular}

The difference between the optimal timestep length in the pilot zones was between 0 and $40 \%$ with a mean value of $16 \%$. That is considered a significant improvement by simple temporary closure of some of the tanks or chambers. Where only one level measurement was available for the DMA, it is not possible to improve uncertainty in this way.

The results show that the uncertainty of water balance calculation is very sensitive to timestep length. In order to avoid unnecessary interpolation, only the whole divisions of 60 minutes were applied in the comparison in Table 3.

Table 3 Uncertainty as a function of timestep length of the pilot zones

\begin{tabular}{cccccccc}
\hline & \multicolumn{7}{c}{ Uncertainty at a given timestep $\left[\mathrm{m}^{3} / \mathrm{h}\right]$} \\
& $\mathrm{t}_{3}$ & $\mathrm{t}_{5}$ & $\mathrm{t}_{10}$ & $\mathrm{t}_{15}$ & $\mathrm{t}_{30}$ & $\mathrm{t}_{60}$ & $\mathrm{t}_{120}$ \\
\hline Zone 1 & 9,8 & $\mathbf{6 , 6}$ & 7,1 & 9,9 & 19,4 & 38,7 & 77,4 \\
Zone 2 & 269,5 & 166,3 & $\mathbf{1 1 5 , 9}$ & 136,0 & 251,4 & 500,2 & 1000,0 \\
Zone 3 & 5,7 & 3,6 & $\mathbf{2 , 7}$ & 3,3 & 6,3 & 12,5 & 25,0 \\
Zone 4 & 12,0 & 7,2 & 3,7 & 2,6 & $\mathbf{2 , 3}$ & 4,0 & 8,0 \\
Zone 5 & 10,0 & 6,1 & $\mathbf{4 , 0}$ & 4,5 & 8,1 & 16,0 & 32,0 \\
Zone 6 & 80,9 & 51,8 & $\mathbf{4 5 , 8}$ & 60,7 & 117,4 & 234,2 & 468,4 \\
\hline
\end{tabular}


Optimal Time Step Length to Minimize Uncertainty of Zonal Water Balance ...A.Bibok and R. Fülöp

The changes of uncertainty due to the temporary closure of some of the tanks or chambers (Table 4) show that the shorter water balance timestep provides lower uncertainty in every zone, except Zone 3. Positive values represent a decrease in uncertainty, thus the lower the better.

Table 4 Change in uncertainty due to shutting out some tanks and chambers

\begin{tabular}{cccccccc}
\hline & \multicolumn{7}{c}{ Change in zonal water balance calculation uncertainty [\%] } \\
& $\mathrm{t}_{3}$ & $\mathrm{t}_{5}$ & $\mathrm{t}_{10}$ & $\mathrm{t}_{15}$ & $\mathrm{t}_{30}$ & $\mathrm{t}_{60}$ & $\mathrm{t}_{120}$ \\
\hline Zone 1 & $12 \%$ & $\mathbf{9 \%}$ & $2 \%$ & $0 \%$ & $0 \%$ & $0 \%$ & $0 \%$ \\
Zone 2 & $59 \%$ & $54 \%$ & $\mathbf{2 3 \%}$ & $7 \%$ & $0 \%$ & $0 \%$ & $0 \%$ \\
Zone 3* & - & - & - & - & - & - & - \\
Zone 4 & $29 \%$ & $29 \%$ & $28 \%$ & $\mathbf{2 4 \%}$ & $7 \%$ & $1 \%$ & $0 \%$ \\
Zone 5 & $29 \%$ & $28 \%$ & $\mathbf{1 5 \%}$ & $5 \%$ & $0 \%$ & $0 \%$ & $0 \%$ \\
Zone 6 & $29 \%$ & $24 \%$ & $\mathbf{7 \%}$ & $2 \%$ & $0 \%$ & $0 \%$ & $0 \%$ \\
\hline
\end{tabular}

* Note that Zone 3 had only one tank and one measurement, thus it was not possible to shut out tank volume in order to improve calculation accuracy

Comparing the uncertainty values with the widely adapted timestep lengths of 30 and 60 minutes produced impressive results (Table 5). The uncertainty can be decreased by $48 \%$ on average compared to 30 minutes timestep length. The comparison between the optimal and the 60 minutes timestep length showed an average of $73 \%$ decrease in uncertainty. Pilot zone 3 has $0 \%$ improvement because of the rounded optimal timestep length equal with the 30 minutes timestep length.

Table 5 Comparison of rounded optimal timestep length with 30 and 60 minutes timesteps

\begin{tabular}{cccccc}
\hline & \multicolumn{2}{c}{ Water balance uncertainty $\left[\mathrm{m}^{3} / \mathrm{h}\right]$} & \multicolumn{2}{c}{ Uncertainty decrease [\%] } \\
& $\mathrm{u}_{\mathrm{topt}}$ & $\mathrm{u}_{\mathrm{t} 30}$ & $\mathrm{u}_{\mathrm{t} 60}$ & $\Delta \mathrm{u}_{30}$ & $\Delta \mathrm{u}_{60}$ \\
\hline Zone 1 & 6,6 & 19,4 & 38,7 & $66 \%$ & $83 \%$ \\
Zone 2 & 115,9 & 251,4 & 500,2 & $54 \%$ & $77 \%$ \\
Zone 3 & 2,7 & 6,3 & 12,5 & $57 \%$ & $79 \%$ \\
Zone 4 & 2,3 & 2,3 & 4,0 & $0 \%$ & $42 \%$ \\
Zone 5 & 4,0 & 8,1 & 16,0 & $50 \%$ & $75 \%$ \\
Zone 6 & 45,8 & 117,4 & 234,2 & $61 \%$ & $80 \%$ \\
\hline
\end{tabular}

Although the inclusion of flow rate dependent uncertainty would make a more robust solution for the problem of zonal water balance calculation uncertainty, no single optimal timestep could have been defined, because the dimension of optimal timesteps increases by the number of flow measurements in the DMA in that case. Equation (6) is utilized best as an indicator to choose sampling rate of measurements in a certain zone. The equation adjusted with flow rate dependent uncertainty can be utilized as a performance indicator for the zonal water balance calculation integrated into the SCADA system.

\section{Conclusion}

Actual uncertainty of water balance calculation is a function of the uncertainty of the flow and level meters, and the flow rate of the flow meters at a given timestep. It is possible to determine an optimal 
calculation timestep length for each district metered area based on the aforementioned properties of the zone. The differences in the $\mathrm{Q}_{1-4}$ values according to Directive 2004/22/EC are neglected in order to provide a scalar instead of a multidimensional space of optimal timestep lengths for every possible combination of flow rates at the meter. The optimal timestep length varied between 7 to 23 minutes in the six observed pilot zones. Although timestep length is usually a whole division of 60 minutes for practical reasons. Considering this, the optimal timestep length varied between 5 to 15 minutes, which is significantly lower than the usually applied 30 or 60 minutes. The adjusted uncertainty equation can be utilized as a performance indicator for the zonal water balance calculation.

Utilizing the result of the research, water balance uncertainty can decrease significantly if it is possible to disconnect some of the chamber or tanks from the zone for a limited time. An example application is the minimum night-flow measurement time window of 01:00-04:00. Water balance and minimum night-flow calculation accuracy can increase without significant investment but by fine-tuning of the post-processing of measurement data.

\section{References}

[1] S. Debiasi, C. M. G. Bort, A. Bosoni, M. Righetti, Influence of Hourly Water Consumption in Model Calibration for Leakage Detection in a WDS, Procedia Engineering 70 (2014) 467-476.

[2] US EPA, Control and mitigation of drinking water losses in distribution systems, United States Environmental Protection Agency, Washington D.C., 2010

[3] P. B. Cheung, G. V. Girol, N. Abe, M. Propato, Night flow analysis and modeling for leakage estimation in a water distribution system, in: Integrating Water Systems - Boxall \& Maksimovic (eds), London, 2010

[4] A. Knobloch, N. Guth, P. Klingel, Automated Water Balance Calculation for Water Distribution Systems, Procedia Engineering 89 (2014) 428-436.

[5] L Hovany, Error in Water Meter Measuring Due to Shorter Flow and Consumption Shorter Than the Time the Meter was Calibrated, $5^{\text {th }}$ International Symposium on Exploitation of Reneweble Energy Sources (2013)

[6] KROHNE Optimflux 4000 Technical datasheet, Online source downloaded on 2018.01.10 at https://www.instrumart.com/assets/krohne_optiflux4000_2012_datasheet.pdf (2016)

[7] Arad Octave MID certification, Online source downloaded on 2018.01.10 at http://arad.co.il/assets/MID-Octave-January-2018.pdf (2018)

[8] Directive 2004/22/EC of the european parliament and of the council, Official Journal of the European Union I. 135 (2004)

[9] M. Farley, Non revenue water - international best practice for assessment, monitoring and control, $12^{\text {th }}$ CWWA Water, wastewater \& Solid Waste Conference, Paradise Island, Bahamas, 2003

[10] Alkasseh, J.M.A., Adlan, M.N., Abustan, I. et al. Water Resour Manage (2013) 27: 1439. https://doi.org/10.1007/s11269-012-0247-2

[11] Kang, Hyeong-Seok \& Kim, Hyunook \& Lee, Jaekyeong \& Lee, Ingyu \& Kwak, Byoung-Youn $\&$ Im, Hyungjoon.. Optimization of pumping schedule based on water demand forecasting using combined model of autoregressive integrated moving average and exponential smoothing. Water Science \& Technology Water Supply. 15. 188-195. (2014) 